\title{
Direct Diastereo- and Enantioselective Vinylogous Michael Additions of Linear Enones
}

\author{
Qunsheng Guo, Americo J. Fraboni, Stacey E. Brenner-Moyer*
}

Department of Chemistry, Rutgers University, 73 Warren Street, Newark, New Jersey 07102 


\title{
Supporting Information
}

\author{
Table of Content
}

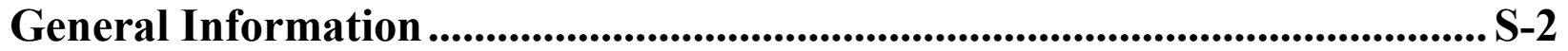

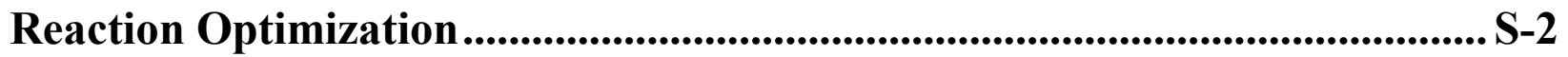

Experimental Procedures .............................................................................. S-4

Compound Characterization Data .......................................................................... S-4

Determination of Relative Stereochemistry ............................................................ S-10

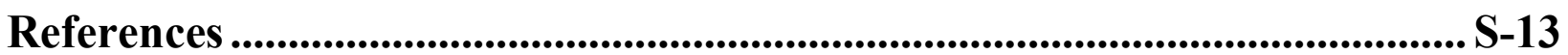


General Information. $\square$ NMR data were acquired on Bruker $500 \mathrm{MHz}$ NMR spectrometers and use the following abbreviations: $\mathrm{s}=$ singlet, $\mathrm{d}=$ doublet, $\mathrm{t}=$ triplet, $\mathrm{m}=$ multiplet, $\mathrm{dd}=\mathrm{doublet}$ of doublets, $\mathrm{ddd}=$ doublet of doublets of doublets, brm = broad multiplet, brs = broad singlet. HRMS spectra were acquired using an MS spectrometer with Q-TOF mass analyzer. Flash chromatography was carried out with F60, 40-63 mm, 60 A silica gel and EMD silica 60 F254 glass TLC plates. Solvents were dried and kept air-free in a solvent purification unit, and were evaporated using a standard rotovapor and high vacuum. All reactions were carried out in ovendried glassware, under an Ar atmosphere. All enals were distilled freshly before use.

\section{Reaction Optimization}

As mentioned in the text, carboxylic acid additives meant to facilitate iminium ion formation were found, instead, to hamper the reaction (entry 1, Table S1). Curiously, whereas the use of $\mathrm{Et}_{3} \mathrm{~N}$ and cinnamic acid additives together doubled the reaction rate (see text), the use of $\mathrm{Et}_{3} \mathrm{~N}$ and benzoic acid additives together hampered the reaction (entry 2). Neat reaction conditions were as effective as $\mathrm{MeOH}$ as solvent (entries 3 and 4). Use of a stronger hydrogen bond donor as solvent did not improve the reaction yield (entry 5). Rather, nonpolar solvents were the most effective for this transformation (entries 6 and 7), being better than halogenated solvents (entries 9-10), and significantly better than polar solvents (entries 3, 5, and 8). Other secondary amine organocatalysts investigated in this reaction generated the product in low yield and/or ee (entries 11-14). 
Table S1. Further optimizations.

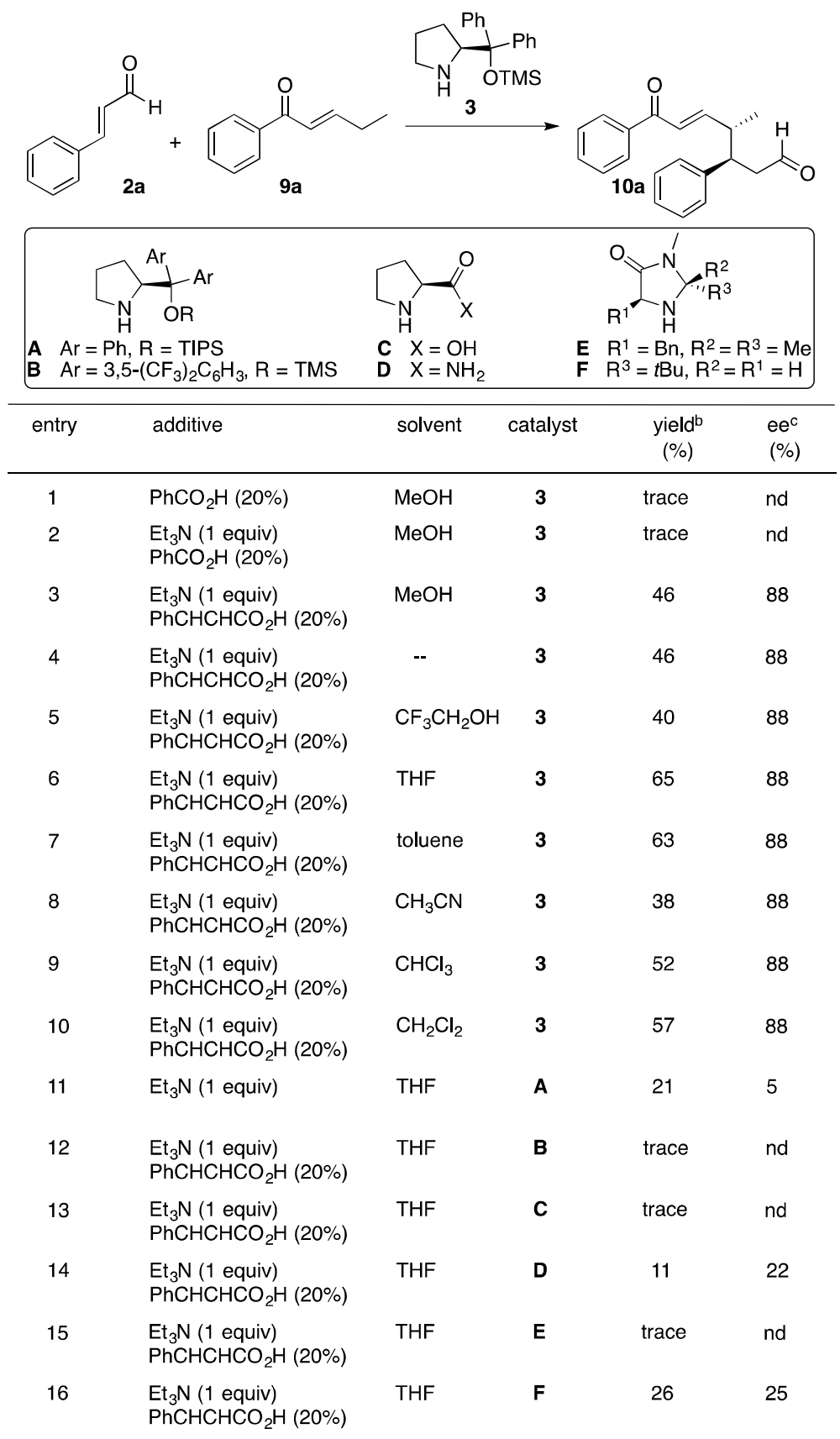

${ }^{\text {a }}$ Reaction conditions: 9a $(0.2 \mathrm{mmol}), \mathbf{2 a}(0.6 \mathrm{mmol})$, catalyst $(0.04 \mathrm{mmol})$, additive, solvent $(0.1 \mathrm{~mL}), \mathrm{rt}, 3 \mathrm{~d} .{ }^{\mathrm{b}}$ Isolated yield of $\mathbf{1 0 a} .{ }^{\mathrm{c}}$ ee determined by chiral phase HPLC. 


\section{Experimental Procedures}

\section{General Experimental Procedure for the Michael Addition Reaction}

To a mixture of the ketone $9(0.20 \mathrm{mmol})$, aldehyde $2(0.60 \mathrm{mmol})$ in THF $(0.1 \mathrm{~mL})$ at r.t. were added Catalyst ent-3 (13.0 mg, $0.004 \mathrm{mmol}, 20 \mathrm{~mol} \%$ ), cinnamic acid (5.9 mg, $20 \mathrm{~mol} \%$ ) and $\mathrm{Et}_{3} \mathrm{~N}(20.2 \mathrm{mg} 0.20 \mathrm{mmol})$. The mixture was further stirred at r.t. for three days. Upon completion, the reaction mixture was directly transferred to a silica gel column and purified by column chromatography (5:1 hexane/ethyl acetate) to afford the Michael addition products.

\section{Compound Charaterization Data}

\section{(E,3S,4R)-4-methyl-7-oxo-3,7-diphenylhept-5-enal (10a)}

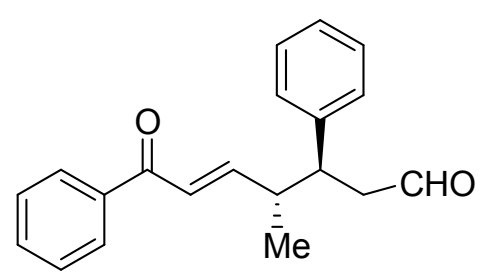

$38 \mathrm{mg}$ colorless oil product, 65\% yield, dr: $>99: 1 ;{ }^{1} \mathrm{H}$ NMR (500 $\left.\mathrm{MHz}, \mathrm{CDCl}_{3}\right): \delta 1.10(\mathrm{~d}, J=6.5 \mathrm{~Hz}, 3 \mathrm{H}), 2.73(\mathrm{dd}, J=7.0,14.0$ $\mathrm{Hz}, 1 \mathrm{H}), 2.84$ (d, $J=7.5 \mathrm{~Hz}, 2 \mathrm{H}), 3.32$ (dd, $J=7.5,14.5 \mathrm{~Hz}, 1 \mathrm{H})$, $6.66(\mathrm{~d}, J=15.5 \mathrm{~Hz}, 1 \mathrm{H}), 6.79(\mathrm{dd}, J=7.0,15.5 \mathrm{~Hz}, 1 \mathrm{H}), 7.14(\mathrm{~d}$, $J=7.0 \mathrm{~Hz}, 2 \mathrm{H}), 7.20(\mathrm{t}, J=7.5 \mathrm{~Hz}, 1 \mathrm{H}), 7.28(\mathrm{t}, J=7.5 \mathrm{~Hz}, 2 \mathrm{H})$, $7.40(\mathrm{t}, J=7.5 \mathrm{~Hz}, 2 \mathrm{H}), 7.51(\mathrm{t}, J=7.5 \mathrm{~Hz}, 1 \mathrm{H}), 7.73(\mathrm{~d}, J=7.5 \mathrm{~Hz}, 2 \mathrm{H}), 9.63(\mathrm{~s}, 1 \mathrm{H}) ;{ }^{13} \mathrm{C} \mathrm{NMR}$ $\left(125 \mathrm{MHz}_{\mathrm{CDCl}}\right): \delta 17.3,42.1,44.7,46.7,126.4,127.0,128.4,128.5,132.6,137.6,140.6$, 150.9, 190.8, 201.1. $v_{\max }$ 3026, 2959, 1718, 1666, 1613, 1444, 1280, 1230, 1013, $980 \mathrm{~cm}^{-1}$. HRMS (ESI): exact mass calculated for $[\mathrm{M}+\mathrm{H}]^{+}\left(\mathrm{C}_{20} \mathrm{H}_{21} \mathrm{O}_{2}\right)$ requires $\mathrm{m} / \mathrm{z} 293.1542$, found $\mathrm{m} / \mathrm{z}$ 293.1537. $[\alpha]_{\mathrm{D}}^{25}=-8.7$ (c 1.0, $\mathrm{CH}_{2} \mathrm{Cl}_{2}, 92 \%$ ee). Enantiomeric excess of the product was measured by chiral stationary phase HPLC analysis using a Chiracel AS-H column (90:10 hexanes $/ i-\mathrm{PrOH}$ at $1.0 \mathrm{~mL} / \mathrm{min}$ ): major enantiomer: $t_{\mathrm{R}}=28.01 \mathrm{~min}$; minor enantiomer: $t_{\mathrm{R}}=37.69$ $\min$.

\section{(E,3S,4R)-4-ethyl-7-oxo-3,7-diphenylhept-5-enal (10b)}

$49 \mathrm{mg}$ pale yellow oil product, 80\% yield, dr: $>99: 1 ;{ }^{1} \mathrm{H} \mathrm{NMR}\left(500 \mathrm{MHz}, \mathrm{CDCl}_{3}\right): \delta 0.87(\mathrm{t}, J=$<smiles>CC[C@H](/C=C/C(=O)c1ccccc1)[C@H](CC=O)c1ccccc1</smiles>
$6.5 \mathrm{~Hz}, 3 \mathrm{H}), 1.23-1.32(\mathrm{~m}, 1 \mathrm{H}), 1.58-1.65(\mathrm{~m}, 1 \mathrm{H}), 2.41-2.47(\mathrm{~m}$, $1 \mathrm{H}), 2.84(\mathrm{dd}, J=4.5,10.5 \mathrm{~Hz}, 2 \mathrm{H}), 3.39(\mathrm{dd}, J=5.5,11.5 \mathrm{~Hz}$, $1 \mathrm{H}), 6.61-6.71(\mathrm{~m}, 2 \mathrm{H}), 7.13(\mathrm{~d}, J=6.0 \mathrm{~Hz}, 2 \mathrm{H}), 7.19(\mathrm{dd}, J=6.5$, 
$12.0 \mathrm{~Hz}, 2 \mathrm{H}), 7.27(\mathrm{t}, J=6.5 \mathrm{~Hz}, 2 \mathrm{H}), 7.41(\mathrm{t}, J=6.0 \mathrm{~Hz}, 2 \mathrm{H}), 7.75(\mathrm{~d}, J=6.5 \mathrm{~Hz}, 2 \mathrm{H}), 9.64(\mathrm{~s}$, $1 \mathrm{H}) ;{ }^{13} \mathrm{C}$ NMR (125 MHz, $\left.\mathrm{CDCl}_{3}\right): \delta 12.0,25.2,43.4,47.5,49.8,127.0,128.1,128.2,128.4$, 128.5, 132.6, 137.6, 140.3, 149.5, 190.4, 201.2. $v_{\max }$ 3026, 2956, 2923, 1716, 1663, 1613, 1449 , 1285, 1247, 1016, $988 \mathrm{~cm}^{-1}$. HRMS (ESI): exact mass calculated for $[\mathrm{M}+\mathrm{Na}]^{+}\left(\mathrm{C}_{21} \mathrm{H}_{22} \mathrm{O}_{2} \mathrm{Na}\right)$ requires $\mathrm{m} / \mathrm{z}$ 329.1517, found $\mathrm{m} / \mathrm{z}$ 329.1521. $[\alpha]_{\mathrm{D}}{ }^{25}=-46.8$ (c $1.0, \mathrm{CH}_{2} \mathrm{Cl}_{2}, 91 \%$ ee). Enantiomeric excess of the product was measured by chiral stationary phase HPLC analysis using a Chiracel AS-H column (90:10 hexanes $/ i-\mathrm{PrOH}$ at $1.0 \mathrm{~mL} / \mathrm{min})$ : major enantiomer: $\mathrm{t}_{\mathrm{R}}=$ $17.24 \mathrm{~min}$; minor enantiomer: $\mathrm{t}_{\mathrm{R}}=19.57 \mathrm{~min}$.

\section{$(R, E)-7-0 x 0-3,7-d i p h e n y l h e p t-5-e n a l ~(10 c)$}

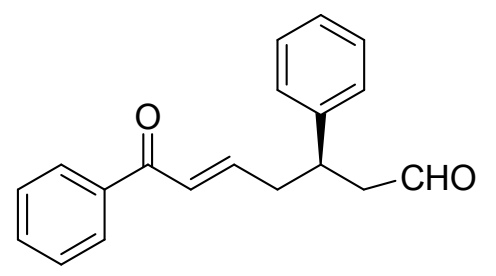

$42 \mathrm{mg}$ colorless oil product, $75 \%$ yield; ${ }^{1} \mathrm{H}$ NMR $(500 \mathrm{MHz}$, $\left.\mathrm{CDCl}_{3}\right): \delta 2.64-2.74(\mathrm{~m}, 1 \mathrm{H}), 2.85(\mathrm{~d}, J=7.0 \mathrm{~Hz}, 2 \mathrm{H}), 3.45-3.51$ (m, 1H), 6.79-6.91 (m, 2H), 7.24-7.32 (m, 3H), $7.35(\mathrm{t}, J=7.5 \mathrm{~Hz}$, $2 \mathrm{H}), 7.45(\mathrm{t}, J=7.5 \mathrm{~Hz}, 2 \mathrm{H}), 7.55(\mathrm{t}, J=7.5 \mathrm{~Hz}, 1 \mathrm{H}), 7.81(\mathrm{~d}, J=$ $7.0 \mathrm{~Hz}, 2 \mathrm{H}), 9.71(\mathrm{t}, J=1.5 \mathrm{~Hz}, 1 \mathrm{H}) ;{ }^{13} \mathrm{C}$ NMR $\left(125 \mathrm{MHz}, \mathrm{CDCl}_{3}\right): \delta 39.1,39.4,49.5,127.0$, 127.3, 128.1, 128.4, 128.8, 132.7, 137.5, 142.4, 145.7, 190.5, 200.8. HRMS (ESI): exact mass calculated for $[\mathrm{M}+\mathrm{H}]^{+}\left(\mathrm{C}_{19} \mathrm{H}_{19} \mathrm{O}_{2}\right)$ requires $\mathrm{m} / \mathrm{z} 279.1385$, found $\mathrm{m} / \mathrm{z} 279.1381 .[\alpha]_{\mathrm{D}}{ }^{25}=+21.7(c$ $1.0, \mathrm{CH}_{2} \mathrm{Cl}_{2}, 98 \%$ ee). Enantiomeric excess of the product was measured by chiral stationary phase HPLC analysis using a Chiracel AS-H column (90:10 hexanes $/ i-\mathrm{PrOH}$ at $1.0 \mathrm{~mL} / \mathrm{min}$ ): major enantiomer: $t_{R}=35.07 \mathrm{~min}$; minor enantiomer: $t_{R}=32.88 \mathrm{~min}$.

\section{$(E, 3 S, 4 R)-7-(4-c h l o r o p h e n y l)-4-m e t h y l-7-o x 0-3-p h e n y l h e p t-5-e n a l ~(10 d)$}

$54 \mathrm{mg}$ colorless oil product, 83\% yield, dr: $>99: 1 ;{ }^{1} \mathrm{H}$ NMR $\left(500 \mathrm{MHz}, \mathrm{CDCl}_{3}\right): \delta 1.14(\mathrm{~d}, J=$

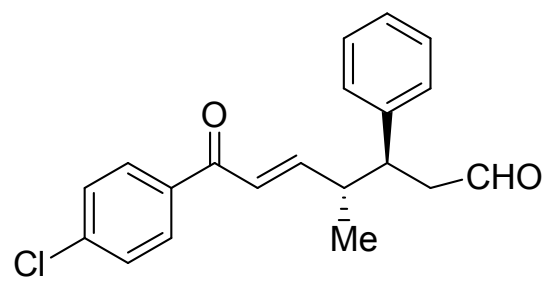

$7.0 \mathrm{~Hz}, 3 \mathrm{H}), 2.77$ (dd, $J=7.0,14.5 \mathrm{~Hz}, 1 \mathrm{H}), 2.87$ (d, $J=7.0 \mathrm{~Hz}$, 2H), 3.34 (dd, $J=7.0,14.0 \mathrm{~Hz}, 1 \mathrm{H}), 6.63(\mathrm{~d}, J=15.5 \mathrm{~Hz}, 1 \mathrm{H})$, $6.81(\mathrm{dd}, J=8.5,15.5 \mathrm{~Hz}, 1 \mathrm{H}), 7.17(\mathrm{~d}, J=7.2 \mathrm{~Hz}, 2 \mathrm{H}), 7.25(\mathrm{dd}$, $J=8.0,15.5 \mathrm{~Hz}, 1 \mathrm{H}), 7.32(\mathrm{dd}, J=2.5,8.0 \mathrm{~Hz}, 2 \mathrm{H}), 7.40(\mathrm{~d}, J=$ $8.0 \mathrm{~Hz}, 2 \mathrm{H}), 7.68(\mathrm{~d}, J=8.0 \mathrm{~Hz}, 2 \mathrm{H}), 9.67(\mathrm{~s}, 1 \mathrm{H}) ;{ }^{13} \mathrm{C} \mathrm{NMR}$ (125 MHz, $\left.\mathrm{CDCl}_{3}\right): \delta 17.5,42.2,44.9,46.9,126.1,127.1,128.5,128.6,128.8,130.0,136.0$, 139.1, 140.7, 151.6, 189.6, 201.1. HRMS (ESI): exact mass calculated for $[\mathrm{M}+\mathrm{H}]^{+}\left(\mathrm{C}_{20} \mathrm{H}_{20} \mathrm{O}_{2} \mathrm{Cl}\right)$ requires $\mathrm{m} / \mathrm{z} 327.1152$, found $\mathrm{m} / \mathrm{z} 327.1150 .[\alpha]_{\mathrm{D}}^{25}=-13.5$ (c $1.0, \mathrm{CH}_{2} \mathrm{Cl}_{2}, 94 \%$ ee). 
Enantiomeric excess of the product was measured by chiral stationary phase HPLC analysis using a Chiracel AS-H column (90:10 hexanes $/ i-\mathrm{PrOH}$ at $1.0 \mathrm{~mL} / \mathrm{min})$ : major enantiomer: $\mathrm{t}_{\mathrm{R}}=$ $20.91 \mathrm{~min}$; minor enantiomer: $t_{R}=27.53 \mathrm{~min}$.

\section{(E,3S,4R)-3-(2-fluorophenyl)-4-methyl-7-oxo-7-phenylhept-5-enal (10e)}

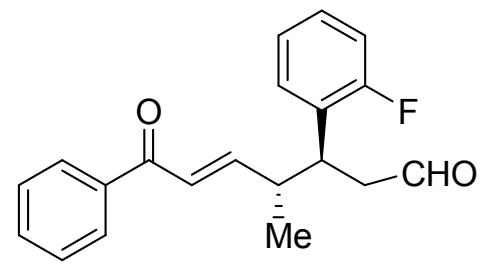

$50 \mathrm{mg}$ pale yellow oil product, $81 \%$ yield, dr: $>99: 1 ;{ }^{1} \mathrm{H}$ NMR $\left(500 \mathrm{MHz}, \mathrm{CDCl}_{3}\right): \delta 1.18(\mathrm{~d}, J=6.5 \mathrm{~Hz}, 3 \mathrm{H}), 2.87(\mathrm{dd}, J=7.5$, $15.0 \mathrm{~Hz}, 1 \mathrm{H}), 2.92(\mathrm{dd}, J=1.0,7.5 \mathrm{~Hz}, 2 \mathrm{H}), 3.68(\mathrm{dd}, J=7.5,15.0$ $\mathrm{Hz}, 1 \mathrm{H}), 6.71(\mathrm{~d}, J=15.5 \mathrm{~Hz}, 1 \mathrm{H}), 6.81(\mathrm{dd}, J=8.0,15.5 \mathrm{~Hz}, 1 \mathrm{H})$, 7.04-7.26 (m, 5H), 7.45 (t, $J=8.0 \mathrm{~Hz}, 2 \mathrm{H}), 7.56$ (t, $J=7.5 \mathrm{~Hz}, 1 \mathrm{H}), 7.76(\mathrm{~d}, J=7.5 \mathrm{~Hz}, 2 \mathrm{H})$, $9.70(\mathrm{~s}, 1 \mathrm{H}) ;{ }^{13} \mathrm{C}$ NMR $\left(125 \mathrm{MHz}, \mathrm{CDCl}_{3}\right): \delta 17.4,38.2,41.3,46.0,115.8(\mathrm{~d}, J=23.0 \mathrm{~Hz}), 124.2$ (d, $J=3.4 \mathrm{~Hz}), 126.4,127.9$ (d, $J=13.8 \mathrm{~Hz}), 128.5$ (d, $J=7.4 \mathrm{~Hz}), 128.6$ (d, $J=8.4 \mathrm{~Hz}), 129.7$ (d, $J=4.6 \mathrm{~Hz}), 132.7,137.6,150.8,159.8,161.8,190.9,200.7$. vmax: 3059, 2967, 1718, 1663 , 1613, 1488, 1449, 1272, 1219, $1013 \mathrm{~cm}^{-1}$. HRMS (ESI): exact mass calculated for $[\mathrm{M}+\mathrm{Na}]^{+}$ $\left(\mathrm{C}_{20} \mathrm{H}_{19} \mathrm{O}_{2} \mathrm{NaF}\right)$ requires $\mathrm{m} / \mathrm{z} 333.1267$, found $\mathrm{m} / \mathrm{z} 333.1270$. $[\alpha]_{\mathrm{D}}{ }^{25}=-10.0\left(c 1.0, \mathrm{CH}_{2} \mathrm{Cl}_{2}, 97 \%\right.$ ee). The product was converted to corresponding ester with $\mathrm{Ph}_{3} \mathrm{PCHCOOMe}$ and enantiomeric excess of the product was measured by chiral stationary phase HPLC analysis using a Chiracel AS-H column (90:10 hexanes $/ i-\mathrm{PrOH}$ at $1.0 \mathrm{~mL} / \mathrm{min})$ : major enantiomer: $\mathrm{t}_{\mathrm{R}}=19.06 \mathrm{~min}$; minor enantiomer: $t_{R}=17.54 \mathrm{~min}$.

\section{$(E, 3 S, 4 R)-3-(4-c h l o r o p h e n y l)-4-m e t h y l-7-o x 0-7-p h e n y l h e p t-5-e n a l ~(10 f)$}

$52 \mathrm{mg}$ colorless oil product, $80 \%$ yield, dr: $>99: 1 ;{ }^{1} \mathrm{H}$ NMR $\left(500 \mathrm{MHz}, \mathrm{CDCl}_{3}\right): \delta 1.13(\mathrm{~d}, J=$

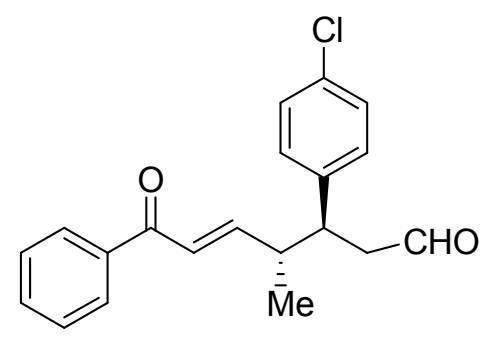

$6.5 \mathrm{~Hz}, 3 \mathrm{H}), 2.75(\mathrm{dd}, J=7.0,14.5 \mathrm{~Hz}, 1 \mathrm{H}), 2.87(\mathrm{~d}, J=7.0 \mathrm{~Hz}$, $2 \mathrm{H}), 3.35$ (dd, $J=6.5,14.5 \mathrm{~Hz}, 1 \mathrm{H}), 6.69-6.83(\mathrm{~m}, 2 \mathrm{H}), 7.12(\mathrm{~d}, J$ $=8.0 \mathrm{~Hz}, 2 \mathrm{H}), 7.30(\mathrm{~d}, J=8.0 \mathrm{~Hz}, 2 \mathrm{H}), 7.46(\mathrm{t}, J=8.0 \mathrm{~Hz}, 2 \mathrm{H})$, $7.57(\mathrm{t}, J=7.5 \mathrm{~Hz}, 1 \mathrm{H}), 7.79(\mathrm{~d}, J=7.5 \mathrm{~Hz}, 2 \mathrm{H}), 9.68(\mathrm{~s}, 1 \mathrm{H}) ;{ }^{13} \mathrm{C}$ NMR (125 MHz, $\left.\mathrm{CDCl}_{3}\right): \delta 17.5,42.0,44.2,46.9,126.6,128.5$, 128.6, 128.7, 129.8, 132.9, 137.6, 139.3, 150.3, 190.6, 200.6. vmax: 3029, 2965, 1718, 1666, 1613, 1491, 1288, 1180, 1086, $1011 \mathrm{~cm}^{-1}$; HRMS (ESI): exact mass calculated for $[\mathrm{M}+\mathrm{Na}]^{+}$ $\left(\mathrm{C}_{20} \mathrm{H}_{19} \mathrm{O}_{2} \mathrm{NaCl}\right)$ requires $\mathrm{m} / \mathrm{z} 349.0971$, found $\mathrm{m} / \mathrm{z}$ 349.0974. $[\alpha]_{\mathrm{D}}{ }^{25}=-14.5$ (c $1.0, \mathrm{CH}_{2} \mathrm{Cl}_{2}, 91 \%$ ee). Enantiomeric excess of the product was measured by chiral stationary phase HPLC analysis 
using a Chiracel AD-H column (90:10 hexanes $/ i$-PrOH at $1.0 \mathrm{~mL} / \mathrm{min})$ : major enantiomer: $t_{\mathrm{R}}=$ $23.62 \mathrm{~min}$; minor enantiomer: $\mathrm{t}_{\mathrm{R}}=27.00 \mathrm{~min}$.

\section{$(E, 3 S, 4 R)-3-(4-m e t h o x y p h e n y l)-4-m e t h y l-7-o x 0-7-p h e n y l h e p t-5-e n a l ~(10 g)$}

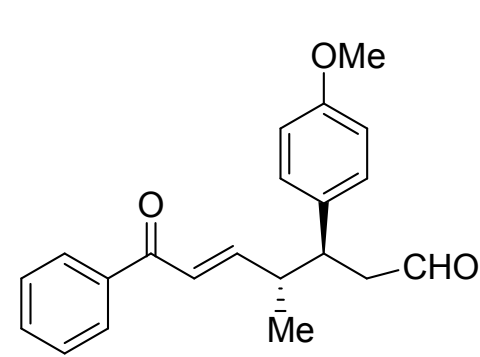

$46 \mathrm{mg}$ pale yellow oil product, 71\% yield, dr: $>99: 1 ;{ }^{1} \mathrm{H}$ NMR $\left(500 \mathrm{MHz}, \mathrm{CDCl}_{3}\right): \delta 1.09(\mathrm{~d}, J=5.5 \mathrm{~Hz}, 3 \mathrm{H}), 2.69(\mathrm{dd}, J=6.0$, $12.0 \mathrm{~Hz}, 1 \mathrm{H}), 2.80(\mathrm{dd}, J=1.5,6.5 \mathrm{~Hz}, 2 \mathrm{H}), 3.27(\mathrm{dd}, J=6.0,11.5$ $\mathrm{Hz}, 1 \mathrm{H}), 3.75(\mathrm{~s}, 3 \mathrm{H}), 6.66(\mathrm{~d}, J=13.0 \mathrm{~Hz}, 1 \mathrm{H}), 6.77-6.83(\mathrm{~m}, 3 \mathrm{H})$, $7.05(\mathrm{~d}, J=7.0 \mathrm{~Hz}, 2 \mathrm{H}), 7.41(\mathrm{t}, J=6.5 \mathrm{~Hz}, 2 \mathrm{H}), 7.52(\mathrm{t}, J=6.0$ $\mathrm{Hz}, 1 \mathrm{H}), 7.75(\mathrm{~d}, J=6.5 \mathrm{~Hz}, 2 \mathrm{H}), 9.63(\mathrm{~s}, 1 \mathrm{H}) ;{ }^{13} \mathrm{C} \mathrm{NMR}\left(125 \mathrm{MHz}, \mathrm{CDCl}_{3}\right): \delta 17.5,42.3,44.2$, 47.1, 55.2, 114.0, 126.4, 128.5, 128.6, 129.4, 132.5, 132.7, 137.7, 151.1, 158.6, 190.8, 201.4. $\nu_{\max }$ :2959, 2926, 1721, 1663, 1610, 1513, 1244, 1183, $1038 \mathrm{~cm}^{-1}$; HRMS (ESI): exact mass calculated for $[\mathrm{M}+\mathrm{Na}]^{+}\left(\mathrm{C}_{21} \mathrm{H}_{22} \mathrm{O}_{3} \mathrm{Na}\right)$ requires $\mathrm{m} / \mathrm{z} 345.1467$, found $\mathrm{m} / \mathrm{z}$ 345.1463. $[\alpha]_{\mathrm{D}}{ }^{25}=-18.5$ (c $1.0, \mathrm{CH}_{2} \mathrm{Cl}_{2}, 93 \%$ ee). Enantiomeric excess of the product was measured by chiral stationary phase HPLC analysis using a Chiracel AS-H column (90:10 hexanes $/ i-\mathrm{PrOH}$ at $1.0 \mathrm{~mL} / \mathrm{min})$ : major enantiomer: $t_{R}=61.16$ min; minor enantiomer: $t_{R}=53.71 \mathrm{~min}$.

\section{(E,3S,4R)-4-methyl-3-(4-nitrophenyl)-7-oxo-7-phenylhept-5-enal (10h)}

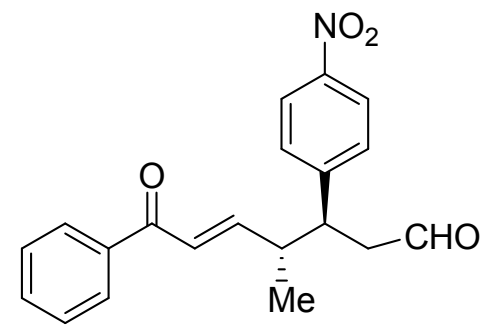

$55 \mathrm{mg}$ yellow oil product, 82\% yield, dr: $>99: 1 ;{ }^{1} \mathrm{H}$ NMR (500 $\left.\mathrm{MHz} \mathrm{CDCl}_{3}\right): \delta 1.12(\mathrm{~d}, J=5.5 \mathrm{~Hz}, 3 \mathrm{H}), 2.78(\mathrm{dd}, J=6.0,11.5$ $\mathrm{Hz}, 1 \mathrm{H}), 2.90-2.99$ (m, 2H), 3.47 (dd, $J=5.5,12.5 \mathrm{~Hz}, 1 \mathrm{H}), 6.70-$ $6.79(\mathrm{~m}, 2 \mathrm{H}), 7.32(\mathrm{~d}, J=7.0 \mathrm{~Hz}, 2 \mathrm{H}), 7.42(\mathrm{t}, J=6.5 \mathrm{~Hz}, 2 \mathrm{H})$, $7.53(\mathrm{t}, J=6.5 \mathrm{~Hz}, 1 \mathrm{H}), 7.77(\mathrm{~d}, J=6.0 \mathrm{~Hz}, 2 \mathrm{H}), 8.14(\mathrm{~d}, J=7.0$ $\mathrm{Hz}, 2 \mathrm{H}), 9.68(\mathrm{~s}, 1 \mathrm{H}) ;{ }^{13} \mathrm{C} \mathrm{NMR}\left(125 \mathrm{MHz}, \mathrm{CDCl}_{3}\right): \delta 17.4,41.6$, 44.3, 46.6, 123.7, 126.6, 128.4, 128.6, 129.3, 133.0, 137.3, 146.9, 148.7, 149.4, 190.0, 199.6. $v_{\max }$ 3031, 2962, 1718, 1666, 1616, 1591, 1519, 1344, 1291, $1008 \mathrm{~cm}^{-1}$; HRMS (ESI): exact mass calculated for $[\mathrm{M}-\mathrm{H}]^{-}\left(\mathrm{C}_{20} \mathrm{H}_{18} \mathrm{NO}_{4}\right)$ requires $\mathrm{m} / \mathrm{z} 336.1236$, found $\mathrm{m} / \mathrm{z} 336.1234$. $[\alpha]_{\mathrm{D}}{ }^{25}=$ 15.3 (c 1.0, $\mathrm{CH}_{2} \mathrm{Cl}_{2}, 96 \%$ ee). Enantiomeric excess of the product was measured by chiral stationary phase HPLC analysis using a Chiracel AS-H column (90:10 hexanes/i-PrOH at 1.0 $\mathrm{mL} / \mathrm{min}$ ): major enantiomer: $\mathrm{t}_{\mathrm{R}}=38.78 \mathrm{~min}$; minor enantiomer: $\mathrm{t}_{\mathrm{R}}=34.98 \mathrm{~min}$. 


\section{(E,3S,4R)-3-(furan-2-yl)-4-methyl-7-oxo-7-phenylhept-5-enal (10i)}

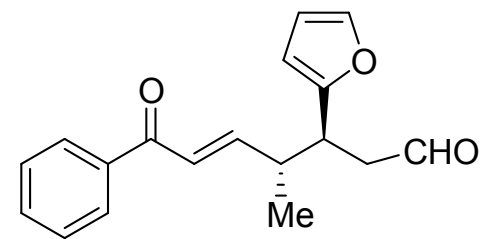

$45 \mathrm{mg}$ yellow oil product, $79 \%$ yield, dr: $>99: 1 ;{ }^{1} \mathrm{H}$ NMR (500 $\left.\mathrm{MHz}, \mathrm{CDCl}_{3}\right): \delta 1.09(\mathrm{~d}, J=6.0 \mathrm{~Hz}, 3 \mathrm{H}), 2.68(\mathrm{dd}, J=4.5,14.0$ $\mathrm{Hz}, 1 \mathrm{H}), 2.78-2.85(\mathrm{~m}, 2 \mathrm{H}), 3.46-3.49(\mathrm{~m}, 1 \mathrm{H}), 6.06(\mathrm{~s}, 1 \mathrm{H}), 6.26$ (s, 1H), 6.76-6.89 (m, 2H), $7.31(\mathrm{~s}, 1 \mathrm{H}), 7.44(\mathrm{t}, J=6.0 \mathrm{~Hz}, 2 \mathrm{H})$, $7.53(\mathrm{t}, J=5.5 \mathrm{~Hz}, 1 \mathrm{H}), 7.85(\mathrm{~d}, J=6.0 \mathrm{~Hz}, 2 \mathrm{H}), 9.67(\mathrm{~s}, 1 \mathrm{H}) ;{ }^{13} \mathrm{C}$ NMR (125 MHz, $\left.\mathrm{CDCl}_{3}\right): \delta$ 17.0, 37.9, 40.5, 44.6, 107.3, 110.1, 126.3, 128.5, 132.7, 137.7, 141.7, 150.6, 154.1, 190.7, 200.5. $v_{\max }$ 2965, 2923, 1718, 1666, 1610, 1438, 1355, 1285, 1219, $1011 \mathrm{~cm}^{-1}$. HRMS (ESI): exact mass calculated for $[\mathrm{M}+\mathrm{Na}]^{+}\left(\mathrm{C}_{18} \mathrm{H}_{18} \mathrm{O}_{3} \mathrm{Na}\right)$ requires $\mathrm{m} / \mathrm{z}$ 305.1154, found $\mathrm{m} / \mathrm{z}$ 305.1160. $[\alpha]_{\mathrm{D}}{ }^{25}=-17.2\left(c 1.0, \mathrm{CH}_{2} \mathrm{Cl}_{2}, 96 \%\right.$ ee). Enantiomeric excess of the product was measured by chiral stationary phase HPLC analysis using a Chiracel AS-H column (90:10 hexanes $/ i-\mathrm{PrOH}$ at $1.0 \mathrm{~mL} / \mathrm{min})$ : major enantiomer: $t_{\mathrm{R}}=21.04 \mathrm{~min}$; minor enantiomer: $t_{R}=28.14$ min.

\section{$(E, 3 S, 4 R)-4-m e t h y l-3-(n a p h t h a l e n-1-y l)-7-0 \times 0-7-p h e n y l h e p t-5-e n a l ~(10 j)$}

$38 \mathrm{mg}$ pale yellow oil product, $55 \%$ yield, $\mathrm{dr}:>99: 1 ;{ }^{1} \mathrm{H} \mathrm{NMR}\left(500 \mathrm{MHz}, \mathrm{CDCl}_{3}\right): \delta 1.19(\mathrm{~d}, J=$<smiles>CC(/C=C/C(=O)c1ccccc1)C(CC=O)c1cccc2ccccc12</smiles>
$7.0 \mathrm{~Hz}, 3 \mathrm{H}), 2.88$ (dd, $J=7.0,14.0 \mathrm{~Hz}, 1 \mathrm{H}), 2.97-3.05(\mathrm{~m}, 2 \mathrm{H})$, $3.53(\mathrm{dd}, J=6.5,15.5 \mathrm{~Hz}, 1 \mathrm{H}), 6.68(\mathrm{~d}, J=15.5 \mathrm{~Hz}, 1 \mathrm{H}), 6.86$ (dd, $J=8.5,15.5 \mathrm{~Hz}, 1 \mathrm{H}), 7.31-7.35(\mathrm{~m}, 3 \mathrm{H}), 7.47-7.52(\mathrm{~m}, 3 \mathrm{H})$, $7.66(\mathrm{t}, J=7.0 \mathrm{~Hz}, 3 \mathrm{H}), 7.83(\mathrm{t}, J=8.0 \mathrm{~Hz}, 3 \mathrm{H}), 9.71(\mathrm{~s}, 1 \mathrm{H}) ;{ }^{13} \mathrm{C}$ NMR (125 MHz, $\left.\mathrm{CDCl}_{3}\right): \delta$ 17.4, 42.1, 45.0, 46.7, 125.8, 126.2, 126.3, 126.7, 127.2, 127.6, 127.7, 128.3, 128.4, 132.5, 133.3, 137.6, 138.2, 150.8, 191.0, 201.0. vmax: 3054, 3031, 2962, 1718, 1666, 1613, 1274, 1216, 1011, $980 \mathrm{~cm}^{-1}$. HRMS (ESI): exact mass calculated for $[\mathrm{M}+\mathrm{Na}]^{+}$ $\left(\mathrm{C}_{24} \mathrm{H}_{22} \mathrm{O}_{2} \mathrm{Na}\right)$ requires $\mathrm{m} / \mathrm{z} 365.1517$, found $\mathrm{m} / \mathrm{z}$ 365.1526. $[\alpha]_{\mathrm{D}}{ }^{25}=-27.6\left(c 1.0, \mathrm{CH}_{2} \mathrm{Cl}_{2}, 87 \%\right.$ ee). Enantiomeric excess of the product was measured by chiral stationary phase HPLC analysis using a Chiracel AS-H column (90:10 hexanes $/ i-\mathrm{PrOH}$ at $1.0 \mathrm{~mL} / \mathrm{min})$ : major enantiomer: $\mathrm{t}_{\mathrm{R}}=$ $33.49 \mathrm{~min}$; minor enantiomer: $t_{\mathrm{R}}=50.03 \mathrm{~min}$.

\section{Compound12}

$23.5 \mathrm{mg}$ colorless oil product, 40\% yield, dr: 82:18; ${ }^{1} \mathrm{H}$ NMR (500 MHz, $\left.\mathrm{CDCl}_{3}\right): \delta 1.09$ (t, $J=$ $6.0 \mathrm{~Hz}, 3 \mathrm{H}), 1.70(\mathrm{~d}, J=5.5 \mathrm{~Hz}, 3 \mathrm{H}), 2.80(\mathrm{ddd}, J=4.5,15.5,42.5 \mathrm{~Hz}, 2 \mathrm{H}), 3.57-3.61(\mathrm{~m}, 1 \mathrm{H})$, 


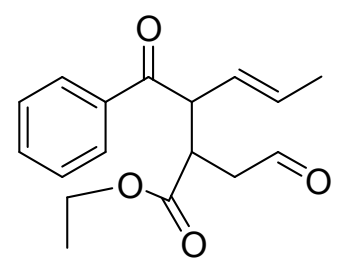

4.00-4.08 (m, 2H), $4.84(\mathrm{t}, J=8.5 \mathrm{~Hz}, 1 \mathrm{H}), 5.18(\mathrm{t}, J=9.0 \mathrm{~Hz}, 1 \mathrm{H}), 5.68-$ $5.73(\mathrm{~m}, 1 \mathrm{H}), 7.44(\mathrm{t}, J=6.5 \mathrm{~Hz}, 2 \mathrm{H}), 7.53(\mathrm{t}, J=6.0 \mathrm{~Hz}, 1 \mathrm{H}), 7.95(\mathrm{~d}, J=$ $6.5 \mathrm{~Hz}, 2 \mathrm{H}), 9.74$ (s, $1 \mathrm{H}) ;{ }^{13} \mathrm{C} \mathrm{NMR}\left(125 \mathrm{MHz}, \mathrm{CDCl}_{3}\right): \delta 13.9,41.1,42.7$, $46.2,53.4,61.3,125.9,128.4,128.6,130.5,133.1,136.7,173.2,199.8$, found $\mathrm{m} / \mathrm{z}$ 311.1262. $[\alpha]_{\mathrm{D}}{ }^{25}=-23.4\left(c 1.0, \mathrm{CH}_{2} \mathrm{Cl}_{2}, 18 \%\right.$ ee $)$. Enantiomeric excess of the product was measured by chiral stationary phase HPLC analysis using a Chiracel AD-H column (98:2 hexanes $/ i-\mathrm{PrOH}$ at $1.0 \mathrm{~mL} / \mathrm{min}$ ): major enantiomer: $\mathrm{t}_{\mathrm{R}}=21.65 \mathrm{~min}$; minor enantiomer: $\mathrm{t}_{\mathrm{R}}=22.78$ $\min$.

\section{Compound13}

$23.5 \mathrm{mg}$ colorless oil product, $75 \%$ yield, dr: 68:32; ${ }^{1} \mathrm{H} \mathrm{NMR}\left(500 \mathrm{MHz}, \mathrm{CDCl}_{3}\right): \delta 1.83(\mathrm{t}, J=$<smiles>C/C=C/C(C(=O)c1ccccc1)C1CC(=O)N(C)C1=O</smiles>
$7.0 \mathrm{~Hz}, 3 \mathrm{H}), 2.69$ (dd, $J=4.5,18.5 \mathrm{~Hz}, 1 \mathrm{H}), 2.93-3.04(\mathrm{~m}, 1 \mathrm{H}), 3.01$ (s, $3 \mathrm{H}), 3.44(\mathrm{t}, J=5.0 \mathrm{~Hz}, 1 \mathrm{H}), 4.96(\mathrm{dd}, J=2.0,10.0 \mathrm{~Hz}, 1 \mathrm{H}), 5.43(\mathrm{t}, J=$ $10.5 \mathrm{~Hz}, 1 \mathrm{H}), 5.79$ (t, $J=8.0 \mathrm{~Hz}, 1 \mathrm{H}), 7.49$ (t, $J=7.5 \mathrm{~Hz}, 2 \mathrm{H}), 7.60$ (t, $J=$ $7.5 \mathrm{~Hz}, 1 \mathrm{H}), 7.95(\mathrm{~d}, J=8.0 \mathrm{~Hz}, 2 \mathrm{H}) ;{ }^{13} \mathrm{C} \mathrm{NMR}\left(125 \mathrm{MHz}, \mathrm{CDCl}_{3}\right): \delta 13.8$, 24.9, 31.7, 41.9, 45.6, 123.3, 128.5, 128.7, 131.1, 133.5, 135.7, 176.8, 178.8, 198.9. HRMS (ESI): exact mass calculated for $[\mathrm{M}+\mathrm{H}]^{+}\left(\mathrm{C}_{16} \mathrm{H}_{18} \mathrm{NO}_{3}\right)$ requires $\mathrm{m} / \mathrm{z}$ 272.1287 , found $\mathrm{m} / \mathrm{z} 272.1284$.

\section{(E,2S,3R)-tert-butyl 2-(formylmethyl)-3-methyl-6-oxo-6-phenylhex-4-enoate (15)}

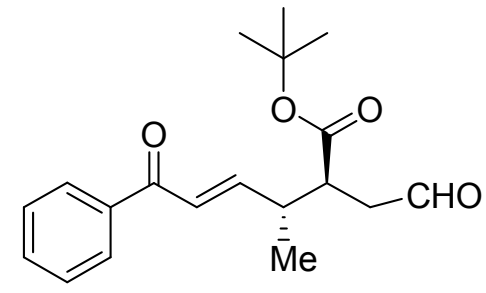

$32 \mathrm{mg}$ colorless oil product, $51 \%$ yield, dr: 83:17; ${ }^{1} \mathrm{H}$ NMR (500 $\left.\mathrm{MHz}, \mathrm{CDCl}_{3}\right): \delta 1.17(\mathrm{~d}, J=7.0 \mathrm{~Hz}, 3 \mathrm{H}), 1.44$ (s, 9H), 2.47 (dd, $J$ $=3.5,17.5 \mathrm{~Hz}, 1 \mathrm{H}), 2.86-2.92(\mathrm{~m}, 2 \mathrm{H}), 2.99-3.03(\mathrm{~m}, 1 \mathrm{H}), 6.88(\mathrm{~d}$, $J=16.5 \mathrm{~Hz}, 1 \mathrm{H}), 6.98(\mathrm{dd}, J=7.0,15.5 \mathrm{~Hz}, 1 \mathrm{H}), 7.49(\mathrm{t}, J=8.0$ $\mathrm{Hz}, 2 \mathrm{H}), 7.58$ (t, $J=7.5 \mathrm{~Hz}, 1 \mathrm{H}), 7.93(\mathrm{~d}, J=7.5 \mathrm{~Hz}, 2 \mathrm{H}), 9.79$ (s,

$1 \mathrm{H}) ;{ }^{13} \mathrm{C}$ NMR $\left(125 \mathrm{MHz}, \mathrm{CDCl}_{3}\right): \delta 15.9,28.0,38.3,42.2,44.4,81.6,125.9,128.6,132.9$, 137.7, 150.4, 172.1, 190.5, 199.9. HRMS (ESI): exact mass calculated for $[\mathrm{M}+\mathrm{Na}]^{+}$ $\left(\mathrm{C}_{19} \mathrm{H}_{24} \mathrm{O}_{4} \mathrm{Na}\right)$ requires $\mathrm{m} / \mathrm{z} 339.1572$, found $\mathrm{m} / \mathrm{z} 339.1564$. $[\alpha]_{\mathrm{D}}{ }^{25}=-6.5\left(c 1.0, \mathrm{CH}_{2} \mathrm{Cl}_{2}, 39 \%\right.$ ee $)$. Enantiomeric excess of the product was measured by chiral stationary phase HPLC analysis 
using a Chiracel AD-H column $(98: 2$ hexanes $/ i-\mathrm{PrOH}$ at $1.0 \mathrm{~mL} / \mathrm{min})$ : major enantiomer: $\mathrm{t}_{\mathrm{R}}=$ 30.58 min; minor enantiomer: $t_{R}=27.95 \min$.

\section{Preparation and charaterization of Compound $14^{1}$}

As per reference 1, to a mixture of catalyst ent-3 $(6.5 \mathrm{mg}, 0.02 \mathrm{mmol}, 10 \mathrm{~mol} \%)$ and Cat. 7 (4.8<smiles>C=CC(C(=O)c1ccccc1)C(CC=O)C(=O)OCC</smiles>
$\mathrm{mg}, 0.01 \mathrm{mmol}, 5 \mathrm{~mol} \%)$ in dry DCE $(1.0 \mathrm{~mL})$ was added unsaturated aldehyde $\mathbf{2 b}(25.6 \mathrm{mg}, 0.2 \mathrm{mmol})$ at room temperature. Then the reaction mixture was heated to $50{ }^{\circ} \mathrm{C}$ and ketones 5 (29 mg, $0.2 \mathrm{mmol}$ ) was added. The reaction mixture was stirred at $50{ }^{\circ} \mathrm{C}$ for 20 hours. Upon completion, the reaction mixture was directly transferred to a silica gel column and purified by column chromatography (5:1 hexane/ethyl acetate) to give $43 \mathrm{mg}$ colorless oil product. $79 \%$ yield, dr: 84:16; ${ }^{1} \mathrm{H}$ NMR (500 MHz, $\left.\mathrm{CDCl}_{3}\right): \delta 1.06(\mathrm{t}, J=6.0 \mathrm{~Hz}, 3 \mathrm{H}), 2.84(\mathrm{ddd}, J=4.0,15.5,53.5 \mathrm{~Hz}$, 2H), 3.58-3.61 (m, 1H), 3.99-4.04 (m, 2H), $4.55(\mathrm{t}, J=8.0 \mathrm{~Hz}, 1 \mathrm{H}), 5.22(\mathrm{~s}, 1 \mathrm{H}), 5.24(\mathrm{~d}, J=3.5$ $\mathrm{Hz}, 1 \mathrm{H}), 5.62-5.68(\mathrm{~m}, 1 \mathrm{H}), 7.43(\mathrm{t}, J=7.0 \mathrm{~Hz}, 2 \mathrm{H}), 7.53(\mathrm{t}, J=6.0 \mathrm{~Hz}, 1 \mathrm{H}), 7.97(\mathrm{~d}, J=6.0 \mathrm{~Hz}$, 2H), $9.73(\mathrm{~s}, 1 \mathrm{H}) ;{ }^{13} \mathrm{C}$ NMR (125 MHz, $\left.\mathrm{CDCl}_{3}\right): \delta$ 14.0, 41.1, 42.7, 52.2, 61.4, 121.6, 128.7, 128.8, 133.3, 134.0, 136.3, 172.9, 199.2, 199.8. HRMS (ESI): exact mass calculated for $[\mathrm{M}+\mathrm{Na}]^{+}\left(\mathrm{C}_{16} \mathrm{H}_{18} \mathrm{O}_{4} \mathrm{Na}\right)$ requires $\mathrm{m} / \mathrm{z} 297.1103$, found $\mathrm{m} / \mathrm{z} 297.1105$.

\section{Determination of relative stereochemistry}<smiles>C[C@H](/C=C/C(=O)c1ccccc1)[C@@H](CC=O)c1ccccc1</smiles>

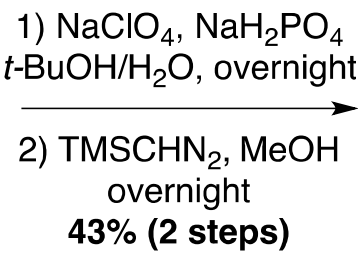<smiles>C[C@@H]1COC(=O)C[C@H]1c1ccccc1</smiles>

1) $\mathrm{NaBH}_{3} \mathrm{CN}, \mathrm{AcOH}$ $\mathrm{MeOH}, 0^{\circ} \mathrm{C}$, 3h

2) $p$ - $\mathrm{TsOH}$, toluene $\Delta$, overnight ref. 2<smiles>COC(=O)C[C@H](c1ccccc1)[C@@H](C)/C=C/C(=O)c1ccccc1</smiles>

1) $\mathrm{OsO}_{4}, \mathrm{NMO}$ acetone $/ \mathrm{H}_{2} \mathrm{O}$ $0{ }^{\circ} \mathrm{C} \rightarrow \mathrm{rt}, 4.5 \mathrm{~h}$

2) $\mathrm{NalO}$ $\mathrm{MeOH} / \mathrm{H}_{2} \mathrm{O}$ $0^{\circ} \mathrm{C}->\mathrm{rt}, 12 \mathrm{~h}$ 18

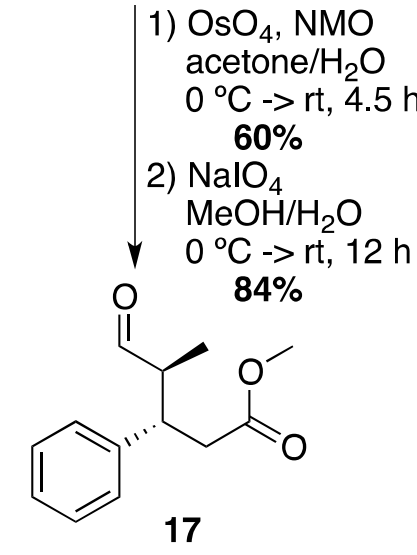


<smiles>C[C@H]1COC(=O)C[C@H]1c1ccccc1</smiles>

\section{Synthesis of (4S,5S)-5-methyl-4-phenyltetrahydro-2H-pyran-2-one 18}

To a solution of $\mathrm{NaClO}_{2}(167.4 \mathrm{mg}, 1.85 \mathrm{mmol})$ and $\mathrm{NaHPO}_{4}(255.4 \mathrm{mg}, 1.85 \mathrm{mmol})$ in $\mathrm{H}_{2} \mathrm{O}(3.3 \mathrm{~mL})$ was added a solution of $\mathbf{1 0 a}(135.3 \mathrm{mg}, 0.46 \mathrm{mmol})$ in $t$ - $\mathrm{BuOH}(6.6 \mathrm{~mL})$. The reaction was stirred at room temperature overnight until complete consumption of 10a, as observed by TLC. The reaction was diluted with brine $(5.0 \mathrm{~mL})$ and extracted with EtOAc $(3 \mathrm{x}$ $10.0 \mathrm{~mL}$ ), then dried over $\mathrm{Na}_{2} \mathrm{SO}_{4}$ and concentrated under reduced pressure to give the crude acid.

The crude acid was dissolved in anhydrous $\mathrm{MeOH}(1.84 \mathrm{~mL})$ and cooled to $0{ }^{\circ} \mathrm{C}$. To the stirred solution was added $\mathrm{TMSCHN}_{2}(920 \mu \mathrm{L}, 1.84 \mathrm{mmol})$ dropwise. The reaction was allowed to slowly warm to room temperature and react overnight. The crude reaction was concentrated by rotary evaporation and purified by flash chromatography (5-7.5\% EtOAc:petroleum ether gradient) to obtain ester 16 in a two-step yield of $43 \%$.

To a solution of ester $16(33.1 \mathrm{mg}, 0.1 \mathrm{mmol})$ in acetone $(710 \mu \mathrm{L})$ and $\mathrm{H}_{2} \mathrm{O}(14 \mu \mathrm{L})$, was added $\mathrm{NMO}\left(50 \%\right.$ in $\left.\mathrm{H}_{2} \mathrm{O}, 62 \mu \mathrm{L}, 0.18 \mathrm{mmol}\right)$, followed by $t$-BuOH $(76 \mu \mathrm{L})$. The solution was cooled to $0{ }^{\circ} \mathrm{C}$ and stirred for 5 minutes. $\mathrm{OsO}_{4}\left(4 \%\right.$ in $\left.\mathrm{H}_{2} \mathrm{O}, 76 \mu \mathrm{L}, 0.012 \mathrm{mmol}\right)$ was added dropwise and the reaction stirred at $0{ }^{\circ} \mathrm{C}$ for 15 minutes. The reaction was brought to room temperature and stirred until complete consumption of 16, as observed by TLC. The reaction was quenched with a $10 \%$ solution of $\mathrm{Na}_{2} \mathrm{~S}_{2} \mathrm{O}_{3}(0.8 \mathrm{~mL})$ and extracted with EtOAc $(3 \times 5.0 \mathrm{~mL})$. The combined organic layers were washed with brine and dried over $\mathrm{Na}_{2} \mathrm{SO}_{4}$. The crude diol was concentrated under reduced pressure and purified by flash chromatography (20\% EtOAc:petroleum ether) to obtain pure diol in $60 \%$ yield.

The diol (21.9 mg, $0.06 \mathrm{mmol})$ was dissolved in $\mathrm{MeOH}(12.9 \mathrm{~mL})$ and $\mathrm{H}_{2} \mathrm{O}(4.3 \mathrm{~mL})$ and cooled to $0{ }^{\circ} \mathrm{C}$. To this solution was added $\mathrm{NaIO}_{4}(161.7 \mathrm{mg}, 0.76 \mathrm{mmol})$ and the reaction was slowly brought to room temperature and stirred until complete consumption of diol, as observed by TLC. The reaction was concentrated under reduced pressure and extracted with diethyl ether ( 3 x $30.0 \mathrm{~mL}$ ). The combined organic phases were dried over $\mathrm{Na}_{2} \mathrm{SO}_{4}$, concentrated, and purified 
by flash chromatography (12.5\% EtOAc:petroleum ether) to obtain pure aldehyde $\mathbf{1 7}$ in $84 \%$ yield.

As per reference 2, to a solution of aldehyde $17(11.4 \mathrm{mg}, 0.05 \mathrm{mmol})$ in anhydrous methanol $(352 \mu \mathrm{L})$ and glacial $\mathrm{AcOH}(118 \mu \mathrm{L})$ at $0{ }^{\circ} \mathrm{C}$, was added $\mathrm{NaBH}_{3} \mathrm{CN}(6.5 \mathrm{mg}, 0.1$ mmol). The reaction was stirred at $0{ }^{\circ} \mathrm{C}$ until full consumption of $\mathbf{1 7}$, as observed by TLC. The reaction was quenched with saturated $\mathrm{NaHCO}_{3}(2.0 \mathrm{~mL})$ and extracted with dichloromethane (3 x $10.0 \mathrm{~mL}$ ). The combined organic phases were dried over $\mathrm{Na}_{2} \mathrm{SO}_{4}$ and concentrated under reduced pressure to obtain the crude alcohol.

As per reference 2, the crude alcohol was dissolved in toluene $(3.24 \mathrm{~mL})$, followed by addition of $p$-TsOH $(1.0 \mathrm{mg}, 0.005 \mathrm{mmol})$. The reaction was heated to $80{ }^{\circ} \mathrm{C}$ and stirred overnight, then cooled to room temperature. To the reaction was added saturated $\mathrm{NaHCO}_{3}(10.0$ $\mathrm{mL})$ and the aqueous layer was extracted with EtOAc $(3 \times 10.0 \mathrm{~mL})$. The organic layers were combined and washed with brine $(30.0 \mathrm{~mL})$, then dried over $\mathrm{Na}_{2} \mathrm{SO}_{4}$ and concentrated under reduced pressure. The crude product was purified by flash chromatography $(17 \%$ EtOAc:petroleum ether) to yield pure lactone 18. $\left.{ }^{2}{ }^{1} \mathrm{H} \mathrm{NMR} \mathrm{(500} \mathrm{MHz,} \mathrm{CDCl}_{3}\right) \delta 7.36$ (t, $J=7.5$ $\mathrm{Hz}, 2 \mathrm{H}), 7.29$ (t, $J=7.0 \mathrm{~Hz}, 1 \mathrm{H}), 7.14(\mathrm{~d}, J=7.5 \mathrm{~Hz}, 2 \mathrm{H}), 4.36(\mathrm{dd}, J=11.5,4.0 \mathrm{~Hz}, 1 \mathrm{H}), 4.11$ (dd, $J=11.0,7.5 \mathrm{~Hz}, 1 \mathrm{H}), 3.35-3.33(\mathrm{~m}, 1 \mathrm{H}), 2.95-2.86(\mathrm{~m}, 2 \mathrm{H}), 2.38-2.37(\mathrm{~m}, 1 \mathrm{H}), 0.83(\mathrm{~d}, J=$ $7.0 \mathrm{~Hz}, 3 \mathrm{H}) \mathrm{ppm} ;{ }^{13} \mathrm{C} \mathrm{NMR}\left(125 \mathrm{MHz}, \mathrm{CDCl}_{3}\right) \delta 170.8,139.5,128.7,128.0,127.4,73.0,41.2$, 33.7, 32.2, 12.5 ppm; HRMS (ESI) $[\mathrm{M}+\mathrm{H}]^{+}$calcd. for $\left[\mathrm{C}_{12} \mathrm{H}_{15} \mathrm{O}_{2}\right]$ 191.1072, found 191.1074.

The relative stereochemistry was determined by examining characteristic peaks in the ${ }^{1} \mathrm{H}$ NMR spectrum as compared to literature data. ${ }^{2}$ The proton corresponding to the doublet of doublets at $4.11 \mathrm{ppm}$ in the cis-lactone is, rather, an apparent triplet at $4.03 \mathrm{ppm}$ in the translactone. Also distinct is the proton that appears as a multiplet at 3.35-3.33 ppm and the two protons that appear as a multiplet at $2.95-2.86 \mathrm{ppm}$ in the cis-lactone. These protons have distinct chemical shifts and splitting patterns relative to the corresponding protons in the translactone, the former appearing as a doublet of doublets at $2.92 \mathrm{ppm}$ and the latter diastereotopically split into a peak at $2.76 \mathrm{ppm}$ and another at $2.66 \mathrm{ppm}$. This evidence led to the conclusion that lactone $\mathbf{1 8}$ is in fact in the cis-configuration, which could be used to determine that the relative stereochemistry of $\mathbf{1 0 a}$ is trans. The absolute stereochemistry at the benzylic carbon of 10a was assigned by analogy with other ent-3 catalyzed conjugate additions. 


\section{References}

1. Gu, Y.; Wang, Y.; Yu, T.-Y.; Liang, Y.-M.; Xu, P.-F. Angew. Chem. Int. Ed. 2014, 53, 14128-14131.

2. Gupta, V.; Sudhir V., S.; Mandal, T.; Schneider, C. Angew. Chem. Int. Ed. 2012, 51, 1260912612. 